\title{
Reflectometry of ice cover at different seasonal conditions by signals of navigation satellites in L1-range
}

\author{
Anatoly Sorokin ${ }^{1 *}$, Evgeniy Kuzmin ${ }^{2}$, Daniil Makarov ${ }^{1}$ \\ ${ }^{1}$ Federal Research Center KSC SB RAS, Krasnoyarsk, Russian Federation \\ ${ }^{2}$ Siberian Federal University, Krasnoyarsk, Russian Federation
}

\begin{abstract}
A series recordings of interference reflectograms from a section of a lake with ice cover in the stage of freezing, stationary winter state, as well as during heating and melting was carried out. The presented experimental data were obtained in L1-range navigation satellites. The interference effects during periods of ice freezing, stationary state of ice (winter) and during melting (spring) were revealed. A variant of volumetric violation of the ice structure as a result of the influence of heat fluxes through the air-ice and ice-water interfaces is considered.
\end{abstract}

\section{Introduction}

The cycle of formation of ice covers of rivers and lakes in Siberia in natural conditions from freeze-up to the complete completion of the ice melting process lasts 5-6 months or more. The practical use of ice covers is quite developed: ice crossings, winter roads, ice on rivers, professional and amateur ice fishing. It is known that in spring the ice cover becomes less durable even with winter thickness, its crystal structure is saturated with water. Thus, the task of non-contact monitoring of the state of ice covers seems to be in demand and urgent. The solution to this problem is advisable using the available global navigation satellite systems (GNSS) GLONASS, GPS and GALILEO. The use of GNSS signals ensures the implementation of continuous monitoring of the earth's cover, atmosphere, and ionosphere. Methods of GNSS reflectometry with long-term registration of amplitude variations of signals from navigation satellites (NS) allows the restoration of the electro physical characteristics of natural environments $[1,2,3]$.

\section{The dynamics of ice cover conditions}

River and lake ice are widely represented on planet Earth. A significant proportion of the areas of water bodies in Siberia in winter is covered with ice, its thickness varies over a wide range. The dynamics of freeze-up on rivers and lakes differs significantly under the same weather conditions. The determining factor for the growth rate of ice thickness is the sum of daily average negative temperatures [4]. The most homogeneous ice in terms of geometrical and electro physical characteristics is formed on small lake water bodies. Under conditions of stable cooling during the freeze-up period, smooth inner and outer ice

${ }^{*}$ Corresponding author: sorav@iph.krasn.ru 
surfaces with a small number of inclusions of gas bubbles and other volume defects are formed. The option of using such objects for studying the processes of interaction of NS signals with ice covers in order to develop a method for diagnosing the mechanical strength of ice is convenient for the practice of relevant experiments.

\section{Dielectric model of ice}

The main radio physical characteristics of fresh water and ice differ. The complex dielectric constant (here in after - CDP) and the refractive index in the range L1 at temperatures of $0^{\circ} \mathrm{C}$ have the following values: dry ice $\varepsilon^{\prime} \sim 3,0-3,2$ and $\varepsilon^{\prime \prime} \sim 0.0003$, water $\varepsilon^{\prime} \sim 86$ and $\varepsilon^{\prime \prime} \sim 7$. These characteristics of wet ice as a mixed dielectric made of ice grains and liquid water at temperatures near $0^{\circ} \mathrm{C}$ interact weakly, which makes it possible to calculate the CDP of the wet ice layer. CDP of wet ice $\varepsilon_{w i}^{\prime}$ is determined by the expression:

$$
\left(\varepsilon_{w i}^{\prime}\right)^{\gamma}=W_{i}\left(\varepsilon_{i}^{\prime}\right)^{\gamma}+W_{\mathrm{w}}\left(\varepsilon_{\mathrm{w}}^{\prime}\right)^{\gamma},
$$

where $\mathrm{W}_{i}, \mathrm{~W}_{w}$ are the volume fractions of ice and water in the water + ice mixture, $\gamma$ is the coefficient, varies in the interval $0<\gamma \leq 1$, is determined experimentally or from theoretical premises.

The real and imaginary parts of the complex values $\varepsilon$ and $\mathrm{n}$ are determined by the relations:

$$
\varepsilon^{\prime}=\alpha^{2}-\beta^{2}, \varepsilon^{\prime \prime}=2 \alpha \beta
$$

Refractive and absorption indices are respectively determined by expressions:

$$
\alpha=\sqrt{ }\left(\sqrt{ }\left(\varepsilon^{\prime 2}+\varepsilon^{\prime \prime 2}\right)+\varepsilon^{\prime}\right) / \sqrt{ } 2 \text { and } \beta=\sqrt{ }\left(\sqrt{ }\left(\varepsilon^{\prime 2}+\varepsilon^{\prime \prime 2}\right) / \sqrt{ } 2\right.
$$

Characteristics of interference reflectograms of ice covers are determined by significant differences between $\alpha$ and $\beta$ of water and ice.

During the life cycle, the temperature field in the volume of the ice cover is dynamic. Temperature gradients at the ice - air - and ice - water boundaries in autumn and spring differ significantly. The heat flux during the freeze-up period from the lower boundary is directed vertically upward and ensures the growth of ice from below. The temperature regime ensures the translation of the $0^{\circ} \mathrm{C}$ temperature point in the ice volume together with the lower boundary during the period of increasing ice cover thickness. During this period, under the condition of a stable decrease in the average air temperature, the ice has a crystalline structure. An increase in air temperature and radiation heating of the upper ice surface in spring smoothly change the direction of the heat flow to the opposite. Localized micro-volumes of liquid water, partially associated with the ice surface, appear in the ice structure. The dielectric constant of surface-bound water decreases markedly in comparison with liquid water. $[5,6]$ In the mixed dielectric refractive model, it is possible to consider wet ice as a three-component medium with a variable concentration of bound water.

\section{Measurements and ice covers}

The registration of interference reflectograms (IR) was carried out according to the traditional scheme [3] based on two types special of GNSS equipment. A set of antennas with linear and right-hand circular polarizations was used. The IR registration scheme is shown in Fig. 1. On the scale of the lake size, the wave front of NS signals is practically 
flat. The direction of its propagation is indicated by the wave vector. An interference field is formed at the point where the antenna is located as a result of the addition of a direct wave and many reflected from the interfaces: air-ice, single reflection and ice-water, with a double passage through the ice mass and the air-ice interface.

The fig. 1 shows the most intense wave. From subsequent reflected waves with a path difference of a shorter coherence length of the NS radiation, the signal powers decrease exponentially, their contribution to the formation of IR is small. At the point where the antenna is located, an interference field is formed as a result of the addition of the direct wave and many reflected ones.

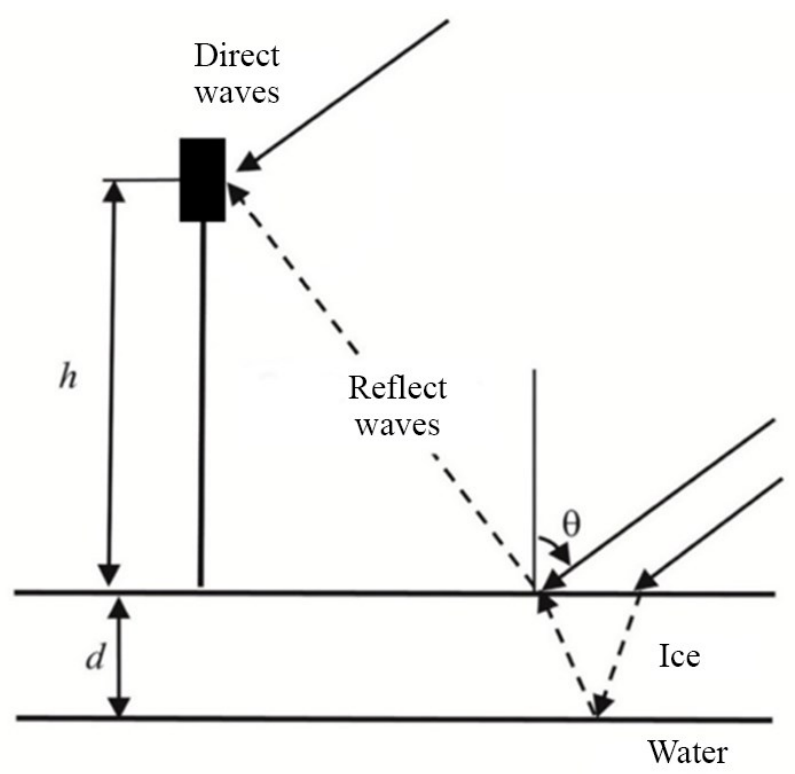

Fig: 1. Scheme of the formation of an interference signal at the receiving antenna

The powers of the signals reflected many times inside the ice significantly decrease; therefore, their contribution to the formation of interference reflectogram is small. Stable interference reflectograms of ice covers were recorded during the period from freeze-up to melting. The trajectories of the navigation satellites and the coordinates of the antenna location make it possible to unambiguously determine the coordinates of the "scanning" center for area reflection on the ice surface. Long-term registration (about 3-4 hour) of the interference reflectogram allows obtaining average data on the local ice thickness

Long-term registration (about 3-4 hour) of the interference reflectogram allows obtaining average data on the local ice thickness [1]. The strength qualities of "spring" ice, besides thickness, are determined by structural changes associated with the appearance of melt water in its volume. The ratio of the real part of the dielectric constants of water and ice near $0^{\circ} \mathrm{C}$ in the L1-range is 26.9 .

Seasonal changes in the strength characteristics of ice sheets are caused by the waterice structural phase transitions. The formation of surface ice occurs rather quickly when the surface water layer is cooled to $0^{\circ} \mathrm{C}$ and there are no waves. Photographs of dry and wet ice samples are shown in Fig. 1, a) and 1, b), respectively. 


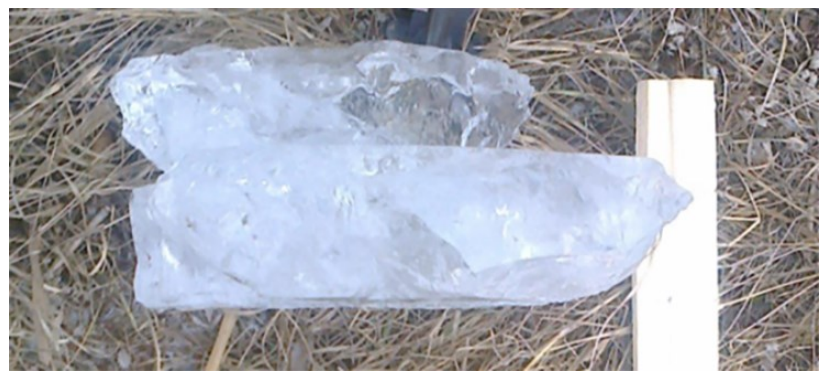

a)

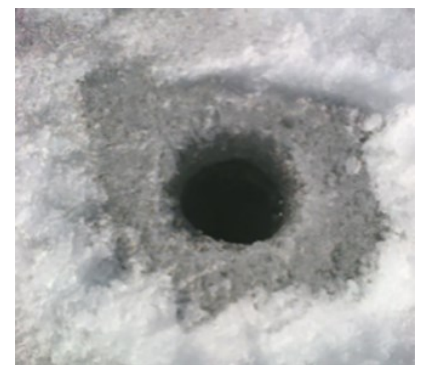

b)

Fig. 2. Photos of ice samples a) newly formed ice (03.11.2018), b) wet ice (31.03.2019)

The visually observed ice cover was a transparent ice layer over the entire surface of the lake with rare extended continuous cracks. According to the classification of lake ice [4], it is lake-water ice, transparent, with air inclusions. In the static state of supercooled water, crystalline needles of ice appear on the surface - the initial forms of crystallization. As the air temperature decreases, the water at the ice-water interface cools and crystallization continues. The ice thickness increases, the initial process of ice needle formation is preserved and fixed in the ice mass. The appearance of local water inclusions during heating is most likely initiated by defects in the crystal structure of polycrystalline ice.

\section{Results and discussion}

A series of IR measurements using signals from the NS GPS and GLONASS in the L1band (1.5-1.6 GHz) was carried out on a lake near Krasnoyarsk with an ice cover in the freeze-up stage (October-November), and in the spring, during the warming up and melting (March-April). A interval of ice thickness varied from $4.2 \mathrm{~cm}$ to $105 \mathrm{~cm}$. Receiving antennas were installed on the ice, or on the coast (during the melting period) at the ice edge. The height of the location of the antennas varied in the interval $3.8 \mathrm{~m}-4.4 \mathrm{~m}$.

In fig. 2 show the IR where $\varphi$ is the elevation angle for ice thicknesses is $4.2 \mathrm{~cm}$ (03.11.2018) and $105 \mathrm{~cm}(31.03 .2019)$, respectively.
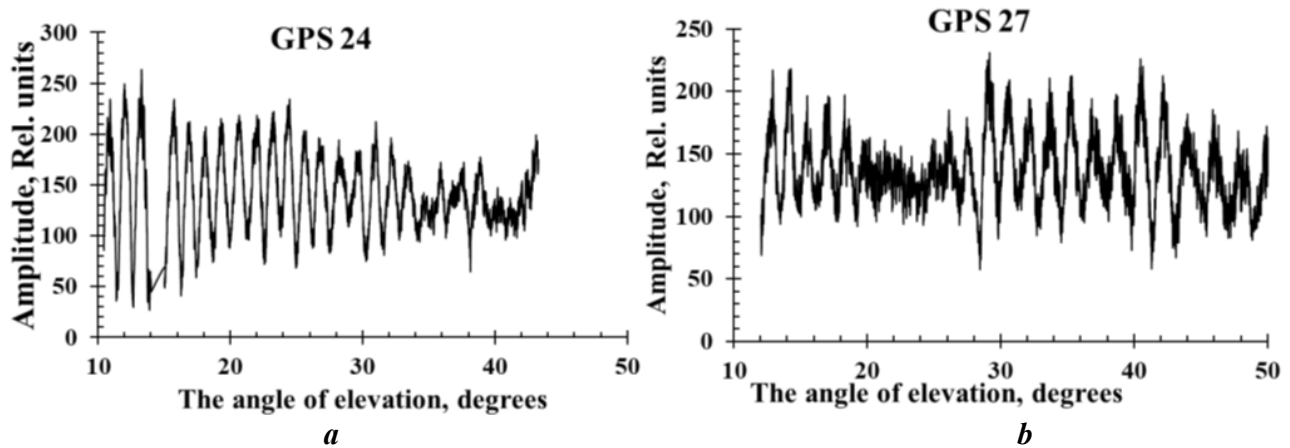

Fig. 2. Experimental IR: a) newly formed ice (03.11.2018); b) wet ice, (31.03.2019)

In Fig. 3 show the experimental IR where ice thicknesses of $64 \mathrm{~cm}(02.04 .2017)$ and $80 \mathrm{~cm}$ (20.04.2019), respectively 

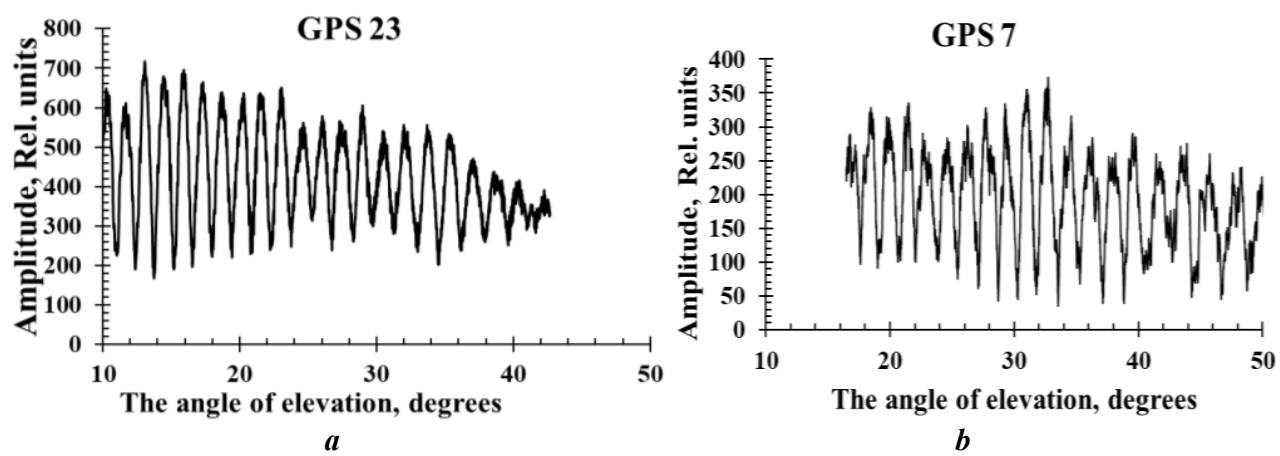

Fig. 3. Experimental IR: a) dry ice, (02.04.2017); b) dry ice + water, (20.04.2019)

As seen from Fig. 2 and 3, experimental IRs have a visually distinguishable modulation structure and noticeable features variation of the amplitude. The parametres IR are determined by the ice thickness and its moisture content. The spatio-temporal the amplitude variations of the total signal are formated by the superposition of the direct and probe signals. Its possible because interaction radio wave with two borders air-ice and ice - water and propogates in ice volume. This process is defined by of signal and depends of the refractive, absorption, thickness and condition of ice surfaces, at the amount and distribution of melt water in its volume.

A model of wet ice as a mixed dielectric can be a three-component medium with a variable concentration of two and more types of water capsules - bulk and surface [7,8]. The temperature field with a vertical gradient in the volume of lake ice forms layers of varying degrees of humidity and thickness. In the freezing stage at a stable negative average daily air temperature, a monolayer model of a rapidly growing dry ice layer is real. At the icewater boundary, a thin layer of wet ice may form, slowly growing with increasing hydrostatic pressure on the lower boundary of the ice cover. When the ice thickness stabilizes and the surface of the air-ice boundary begins to warm up, an upper layer of wet ice grows downwards. For some time, three layers co-exist in the ice thickness: the growing wet upper and lower layers, and the decreasing dry middle layer

In IR processing, it is necessary to take into account the influence of coherence length of proube signals. The probing signals of the NS GPS and GLONASS in the frequency range 1.5 -- $1.6 \mathrm{GHz}$ ), have similar values of the signal power in the near-Earth space, the type of modulation, and the signal repetition period. The differences lie in the method of separating the signals of individual satellites and the width (signal frequency bands: $\sim 2 \mathrm{MHz}$ for GPS and $\sim 1 \mathrm{MHz}$ for GLONASS). Accordingly, the coherence lengths are: GPS $\sim 300 \mathrm{~m}$, GLONASS $\sim 600 \mathrm{~m}[1,2]$.

\section{Conclusions}

A principal possibility of the method for monitoring the state of ice covers is due to a significant difference in the complex permittivity of water and ice. The presence of melt water in the ice structure determines the refractive characteristics of ice and the parameters of experimental interference reflectograms. The process of ice formation and melting is determined by vertical heat flows through the air-ice and ice-water boundaries. A layered refractive model adapted to the ice life cycle is promising in data processing algorithms.

The use of GNSS specialized equipment provides registration of amplitude variations of navigation satellite signals for several hours and obtaining the corresponding array of data on the state of ice. 


\section{References}

1. S. Jin, E., Cardellach F.Xie GNSS Remote Sensing. (New York, London: Springer Dordrecht Heidelberg, 2014)

2. D. S.Makarov, D. V.Kharlamov A. V. Sorokin Siberian Journal of Science and Technology. Vol. 20, 1 (2019), Doi: 10.31772/2587-6066-2019-20-1-8-19

3. M.I. Mikhaylovm, K.V. Muzalevsry Mjronov V.L, Modern problems of remote sensing of the Earth from space. 14, 2, pp 167-174.

4. V.V. Bogorodsky, V.P.Gavrilo, Ice. Physical properties. Modern methods of glaciology. (Gidrometeoizdat, Leningrad, 1980)

5. Yu.I. Lukin, V. L. Mironov, S. A. Komarov, Izvestiya vuzov. Physics, 51, 9 (2008)

6. V.L. Mironov, Yu.I. Lukin, Izvestiya vuzov. Physics, 53, 9 (2010)

7. G.S. Bordonsky, A.O.Orlov, Letters to ZhTF, 45, 5 (2019)

8. Nilsson, L.G.M. Pettersson, Chemical Physics 389 (2011) 